ANL-HEP-PR-10-2

January 10,2010

May 20, 2010 (updated)

\title{
Searches for TeV-scale particles at the LHC using jet shapes
}

\author{
S.V. Chekanov and J. Proudfoot \\ HEP Division, Argonne National Laboratory, 9700 S.Cass Avenue, \\ Argonne, IL 60439 USA
}

\begin{abstract}
New particles at the TeV scale can decay hadronically with strongly collimated jets, thus the standard reconstruction methods based on invariantmasses of well-separated jets can fail. We discuss how to identify such particles in $p p$ collisions at the LHC using simple jet shapes which help to reduce the contribution of QCD-induced events. We focus on a rather generic example $X \rightarrow t \bar{t} \rightarrow$ hadrons, with $X$ being a heavy particle, but the approach is well suited for reconstruction of other decay channels characterized by a cascade decay of known states.
\end{abstract}




\section{Introduction}

Proton-proton collisions at the LHC will allow physicists to search for new $\mathrm{TeV}$ mass particles that can corroborate or disprove current theories such as the Standard Model (SM). One promising path to discoveries at the LHC is through modelindependent searches in which events can be classified in exclusive classes according to the number of identified high- $p_{T}$ objects, such as jets and leptons. Such event classes can be analyzed by reconstructing event rates, invariant masses and other event characteristics. However, heavy particles with masses close to the TeV scale can decay into more than two states which undergo a significant Lorentz boost. This leads to partial or complete overlap of the decay products which cannot be reconstructed as separate objects. In the case of jets, this closes the opportunity of bump hunting in invariant-mass spectra using individual jets since the event signatures will be indistinguishable from that of the standard QCD-induced events.

One approach to tackle this problem is to use jet shapes (see recent publications [1-11]). One hopes that its distinct characteristics can be useful for reduction of the overwhelming rate of conventional QCD jets, thus opening the path to a direct observation of new states.

In this paper we will discuss how to reconstruct a rather generic decay $X \rightarrow t \bar{t}$, (with $X$ being a particle at the $\mathrm{TeV}$ scale) using jet-shape variables. The decay channel involving the production of top quarks is particularly important since they are expected to be strongly coupled to the electroweak symmetry breaking sector, thus the channel has a large potential for discovery of new states at the LHC. Examples of high-mass resonances decaying predominantly to $t \bar{t}$ have recently been discussed in [1, 2, 12, 13]. Additional channels include technicolor models, strong electroweak symmetry breaking models, topcolor, SM Higgs, MSSM Higgs etc. (see [14] for a review).

Recently, a detailed discussion of how to reject QCD background using monojets (jets that fully contain decay products of top quarks) has been discussed within the model with the lightest Kaluza-Klein $(\mathrm{KK})$ excitation of the gluon $g^{(1)} \rightarrow t \bar{t}$ in Randall-Sundrum scenario [1,2, 15. It was pointed out that jet substructure can be useful to increase the signal-to-background ratio since more conventional approaches will fail due to the large overlap of decay products. For example, $b$-tagging may be inefficient because of a large boost of $b$-quarks from top decays and, therefore, a significant collimation of the decay products from the $b$ decays. It was concluded that extraction of the KK signal requires a background rejection of about a factor $10[2]$.

In this paper we will extend the studies presented in Ref. [2], focusing on jet shapes for rejection of QCD background in the hadronic decay $X \rightarrow t \bar{t}$. We will show that using only jet-shape variables, one can easily reduce the contribution to the jet-jet invariant masses from the standard QCD by more than one hundred, with only a factor three reduction for the signal events. Thus we confirm a rather good perspective on searches for heavy-mass particles in the $t \bar{t}$ decay channel, or any other 
similar decay channel with a cascade decay of known states. In addition, we extend the reconstruction aspect of the previous paper [2] in several areas: (a) We will consider the anti- $k_{T}$ algorithm [16] which is used for the default jet reconstruction at the ATLAS experiment; (b) we will study the jet-shape variables and calculate correlations between them; (c) using the jet shapes for background rejection, we will estimate a minimal cross section necessary for $6 \sigma$ observation of a new $\mathrm{TeV}$-scale particle using the dijet invariant-mass signatures and $200 \mathrm{pb}^{-1}$ of $p p$ collisions at $10 \mathrm{TeV}$. We argue that the approach allows to obtain a similar discovery-level cross section that was previously anticipated for $10 \mathrm{fb}^{-1}$ of $p p$ collision at $14 \mathrm{TeV}$ [17].

\section{Jet shapes for searches of $\mathrm{TeV}$-scale particles}

The idea of reconstruction of top quarks using monojets, i.e. collecting all energy deposits in a large cone around the top-quark direction, has previously been discussed (see, for example, [17]). A new approach to this problem is to use jet shapes to reject QCD background as recently discussed in several publications (see, for example, the most recent papers [3-11]).

Before going into a detailed description of the jet-shape approach, we recall a few basic kinematic features when dealing with decays of heavy particles. If a TeV-mass particle $X$ decays into several known particles without subsequent cascade decay, then their decay products are spatially separated. For example, a heavy graviton decaying into $\gamma \gamma$ should not cause reconstruction problems since the photons are produced back-to-back in the laboratory frame. The situation is dramatically different for a cascade-type of decays; due to a significant Lorentz boost, the decay products can easily overlap. This is illustrated in Fig. 1 which shows the largest angular separation between three quarks originating from the same parent top quark in the reaction $X \rightarrow t \bar{t} \rightarrow W^{+} b_{1} W^{-} b_{2}$, with subsequent decays of the $W$ bosons into two quarks, $W \rightarrow q_{1} q_{2}$. The figure was obtained using a simple Monte Carlo simulation which consists of two steps: 1) a calculation of decays in the center-ofmass frame of daughter particles, assuming flat distributions of the azimuthal and polar angles in this frame; 2) performing a Lorentz boost of daughter particles to the laboratory frame. The original momentum of the $X$ particle does not affect the angles between $q_{i}$ quarks and the top quark, while the mass of the state $X$ has a significant impact on the angular distribution. A similar distribution can be obtained for simpler decays, such as $X \rightarrow W^{+} W^{-} \rightarrow 4 q$.

The above observation has a direct implication for experimental searches of heavy particles. The $k_{T}$ jet algorithm [18] with the size $R=0.4$ collects energy deposits into a single jet if angular separation between them is not larger than $21^{\circ}$; thus two jets cannot be resolved as two separate objects if jet centers are separated by an angle smaller than this angle. In the case of the size $R=0.6$, the minimal angular 


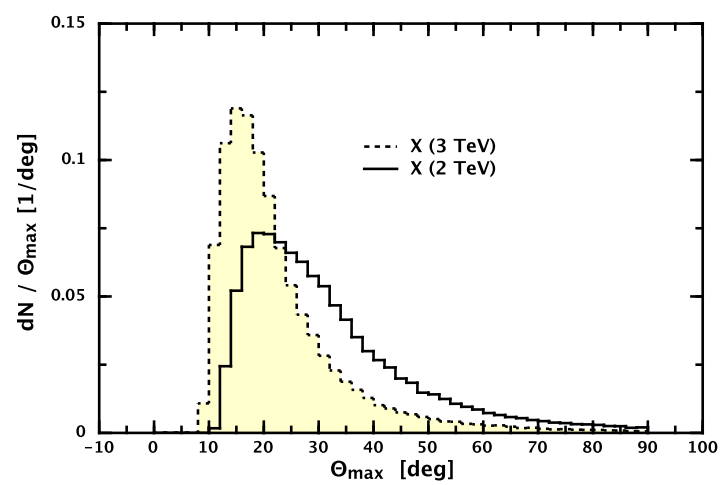

Figure 1: A Monte Carlo simulation of the probability distribution for the maximum angle between quarks $(q, b)$ coming from the parent top quark in the decay $X \rightarrow t \bar{t}$, with $t \rightarrow W b \rightarrow q \bar{q} b$. The simulation consists of a calculation of the decay angles in the center-of-mass frame of the daughter particles and subsequent Lorentz boost to the laboratory frame. The original momentum of the particle $X$ was set to 10 $\mathrm{GeV}$, but the distributions shown depend only on its mass, which was set to $3 \mathrm{TeV}$ (dashed line) or $2 \mathrm{TeV}$ (continues line).

separation is $31^{\circ}$. A similar conclusion applies to cone algorithms ${ }^{1}$. Figure 1 shows that a significant fraction of events contain decay products separated by angles less than $21^{\circ}\left(31^{\circ}\right)$. In the case of $3 \mathrm{TeV}$ particles, the largest fraction of jets will overlap and will not be reconstructed as separate objects. A similar conclusion was obtained in $[2]$.

As mentioned before, we will use the anti- $k_{T}$ version [16] of the popular $k_{T}$ algorithm [18]. Our strategy for identification of jets from top quarks produced by $\mathrm{TeV}$-scale particles will be based on three jet-shape variables. In the case of fully overlapping jets, one should expect two energetic jets, each of which is due to the hadronic decay of a top quark. Thus we will be interested in the shapes of two jets leading in transverse momentum $\left(p_{T}\right)$. The following jet-shape variables will be considered: the jet mass, the jet width and the eccentricity parameter. The jet mass calculated as the mass of all constituents inside a jet, and the jet width and jet eccentricity are considered below:

\section{$2.1 \quad$ Jet width}

The jet width is defined as the $p_{T}$ weighted sum of jet constituents falling into the ring $d R$ in pseudorapidity $\eta$ and azimuthal angle $\phi$ centered with respect to the jet

\footnotetext{
${ }^{1}$ This is not an exact statement, since clustering for the $k_{T}$ and for the conventional cone algorithm are done differently assuming the same input jet-size parameter.
} 
axis. Mathematically, it can be written as :

$$
\frac{\sum_{i=1}^{N} d R^{i} p_{T}^{i}}{\sum_{i}^{N} p_{T}^{i}}
$$

where $d R^{i}=\sqrt{d \phi_{i}^{2}+d \eta_{i}^{2}}$ defines a ring positioned at the jet center, with $d \phi_{i}$ and $d \eta_{i}$ being distances in the azimuthal angle and pseudorapidity of a jet constituent $i$ with a transverse momentum $p_{T}^{i}$ from the jet center. The sum runs over all particles $N$ inside the jet.

\subsection{Jet eccentricity}

The jet eccentricity, ECC, is defined as $1-v_{\max } / v_{\min }$, where $v_{\max }\left(v_{\min }\right)$ is the maximal (minimal) values of variances of jet constituents along the principle (minor) axis. The calculation consists of a few steps: First, for each jet constituent $i$ with the energy $e_{i}$, the energy-weighted centers in $\eta$ and $\phi$ are calculated as:

$$
\bar{\phi}=\sum_{i=1}^{N} d \phi_{i} e_{i} / \sum_{i=1}^{N} e_{i}, \quad \bar{\eta}=\sum_{i=1}^{N} d \eta_{i} e_{i} / \sum_{i=1}^{N} e_{i},
$$

where $d \phi_{i}$ and $d \eta_{i}$ are defined as for the jet width in Eq. (1). The differences from the energy-weighted centers are given by $\Delta \eta_{i}=\eta_{i}-\bar{\eta}$ and $\Delta \phi_{i}=\phi_{i}-\bar{\phi}$. Then, the standard principle-component analysis [19] was performed to determine the axis along which the energy-weighted variance of jet constituents is maximized (principle axis), while the orthogonal axis was defined as the minor axis. The rotation angle $\theta$ of the ellipse populated by hadrons inside a jet is given by:

$$
\tan (2 \theta)=\frac{2 \sum_{i=1}^{N} e_{i} \Delta \eta_{i} \Delta \phi_{i}}{\sum_{i=1}^{N} e_{i}\left(\Delta \phi_{i}^{2}-\Delta \eta_{i}^{2}\right)}
$$

while the angle of the orthogonal axis is shifted by $\pi / 2$ with respect to $\theta$. The energy-weighted variances $v_{1}$ and $v_{2}$ for the axes are calculated as:

$$
\begin{aligned}
& v_{1}=N^{-1} \cdot \sum_{i=1}^{N} e_{i}\left(\cos (\theta) \Delta \eta_{i}-\sin (\theta) \Delta \phi_{i}\right)^{2}, \\
& v_{2}=N^{-1} \cdot \sum_{i=1}^{N} e_{i}\left(\sin (\theta) \Delta \eta_{i}+\cos (\theta) \Delta \phi_{i}\right)^{2} .
\end{aligned}
$$

Finally, the largest value of the variance is assigned to $v_{\max }$, while the smallest to $v_{\text {min }}$. The jet eccentricity ranges from 0 (for perfectly circular jets) to 1 (for an infinitely elongated jet shape). This parameter is similar to $\operatorname{det} S^{\perp}[15$ ] discussed within the context of possible strategies to identify boosted top quarks. A similar parameter (the so-called "planar flow" parameter) was also discussed in Ref. [5].

We did not use the jet substructure variables, such as YSplitter values [3], which define scales at which a single $k_{T}$ jet flips into two and three subjets. For the anti- $k_{T}$ algorithm, these parameters are expected to be sensitive to soft subjets and thus can be strongly affected by pileup and jet-reconstruction effects. 


\begin{tabular}{|c|c|c|c|}
\hline & mass & width & ECC \\
\hline mass & 1 & $0.88(0.78)$ & $0.32(-0.10)$ \\
width & $0.88(0.78)$ & 1 & $0.34(-0.11)$ \\
ECC & $0.32(-0.10)$ & $0.34(-0.11)$ & 1 \\
\hline
\end{tabular}

Table 1: Pearson's correlation coefficients for the jet-shape variables calculated for inclusive $p p$ events and events with $Z^{\prime} \rightarrow t \bar{t} \rightarrow 6 q$, where $Z^{\prime}$ has the mass $2 \mathrm{TeV}$ (shown in parenthesis). In both cases the events are generated with the PYTHIA model. The correlation coefficients are defined as the covariance between $i$ th and $j$ th jet-shape variable, divided by the products of the corresponding standard deviations. In the case of fully correlated variables, the correlation coefficient is one, while it is zero in the case of uncorrelated variables. The correlation matrix was obtained for anti- $k_{T}$ jets with transverse momenta larger than $500 \mathrm{GeV}$.

\subsection{Studies of correlations}

The analysis of the jet shapes was performed using the PYTHIA Monte Carlo model [20] included in the RunMC package [21] which interfaces FORTRAN Monte Carlo models with ROOT [22] and other $\mathrm{C}++$ libraries. Jets and their shapes were reconstructed using the FastJet package [23].

One should note that the jet shape variables are not totally independent of each other. This was checked by running the PYTHIA Monte Carlo model for fully inclusive $p p$ production at $10 \mathrm{TeV}$ and reconstructing the jet shapes for leading jets in $p_{T}$. A minimum jet transverse momentum $500 \mathrm{GeV}$ was required. Table 1 shows the Pearson's correlation coefficients used to estimate the strength of the linear relationship between the jet-shape variables. We remind the reader that if two observables are strongly correlated, the corresponding correlation coefficient is close to unity. Table 1 shows that jet widths correlate with masses. The jet eccentricity weakly correlates with other variables, which implies that an additional rejection can be obtained when using this variable. This conclusion also holds for fully hadronic decays of $Z^{\prime} \rightarrow t \bar{t} \rightarrow 6 q$ where $Z^{\prime}$ has the mass $2 \mathrm{TeV}$, although now some variables show a weak anti-correlation.

\section{Searches using jet shapes}

As stated earlier, we are interested in the jet shapes for the decay $X \rightarrow t \bar{t} \rightarrow$ $W^{+} b_{1} W^{-} b_{2}$, with $X$ being a particle with a mass close to the TeV scale and $W \rightarrow$ $q_{1} q_{2}$. We will attempt to reconstruct the mass of the state $X$ but, more importantly, we will try to disentangle the hadronic final-state signatures of this decay from those of the standard QCD jets using the jet shapes defined in Section 2.

We simulated heavy-particle decays using $Z^{\prime}$ bosons as they are included in the PYTHIA model, forcing these states to decay to $t \bar{t}$ pairs. Both top quarks were 

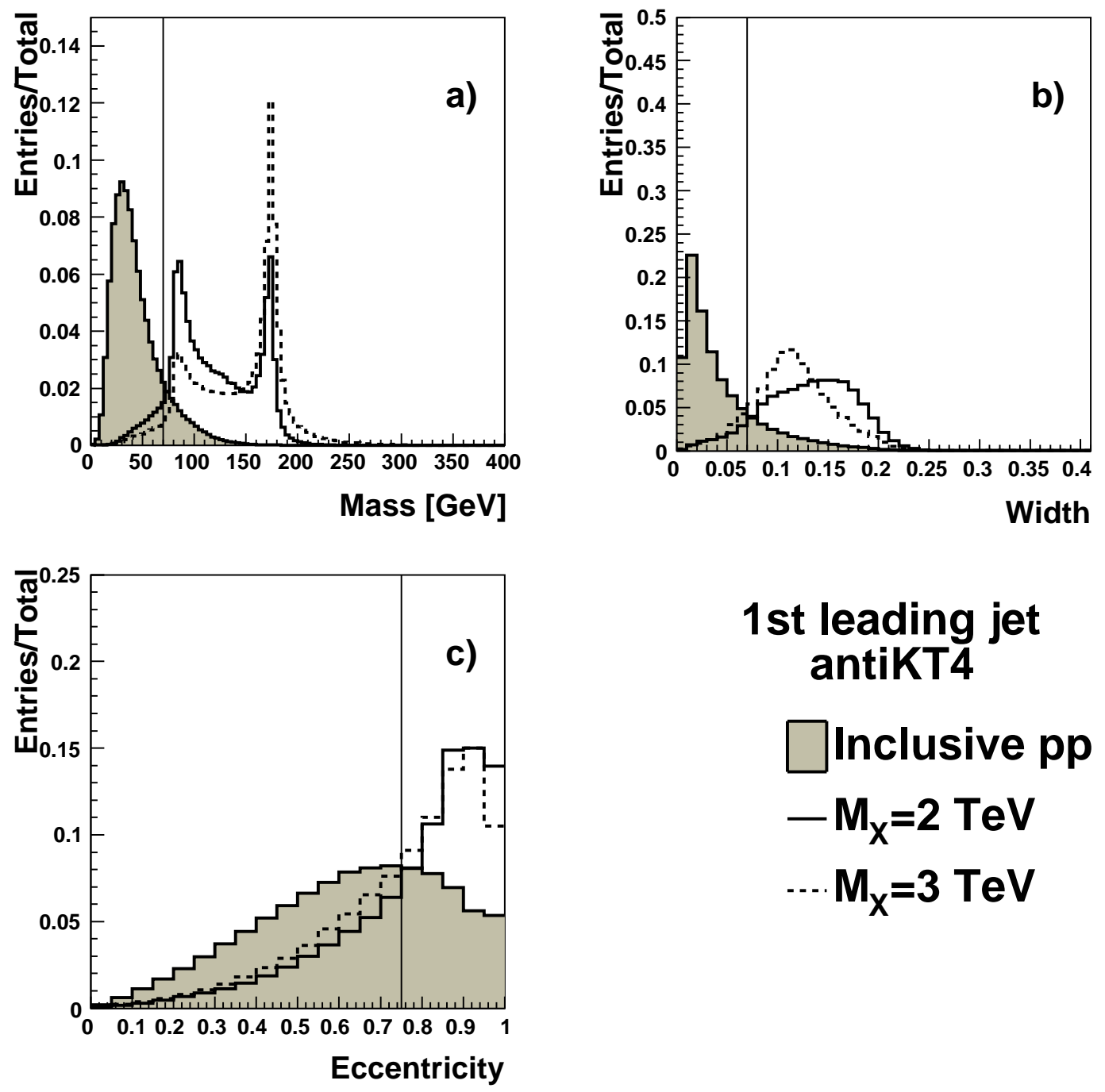

\section{1st leading jet antiKT4} Inclusive pp $-\mathrm{M}_{\mathrm{x}}=2 \mathrm{TeV}$ $\cdots M_{x}=3 \mathrm{TeV}$

Figure 2: Jet shape variables for the leading jet in $p_{T}$ for inclusive $p p$ collisions (filled histograms) simulated with the PYTHIA model. Also shown are the shape variables for $X \rightarrow t \bar{t} \rightarrow W^{+} b_{1} W^{-} b_{2}$, with $W$ bosons hadronically decaying into two jets. The state $X$ was simulated using a $Z^{\prime}$ particle with the mass of 2 and $3 \mathrm{TeV}$ (solid and dashed lines, respectively). Events were selected with at least one jet with $p_{T}>500 \mathrm{GeV}$ using the anti- $k_{T}$ jet algorithm. The vertical lines show the cuts applied to reject inclusive QCD events. 


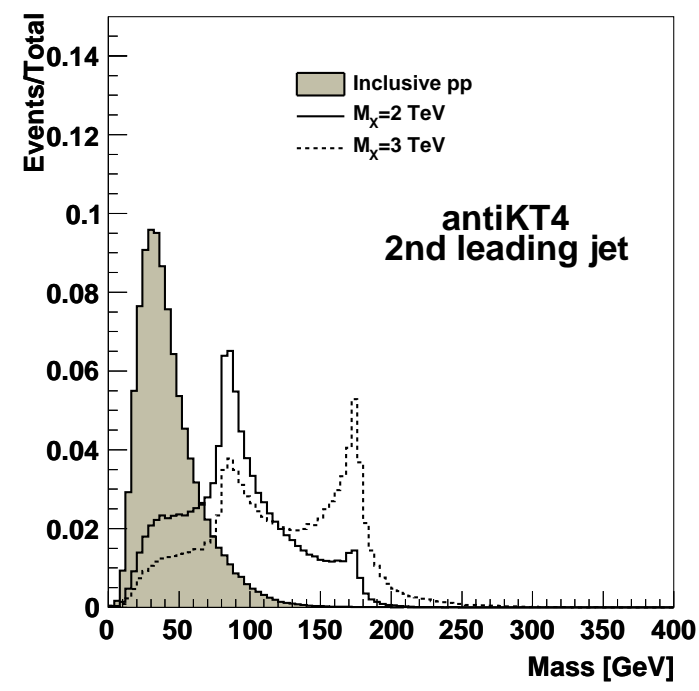

Figure 3: The jet masses for the second leading jets in $p_{T}$ reconstructed using the anti- $k_{T}$ jet algorithm with $R=0.4$.
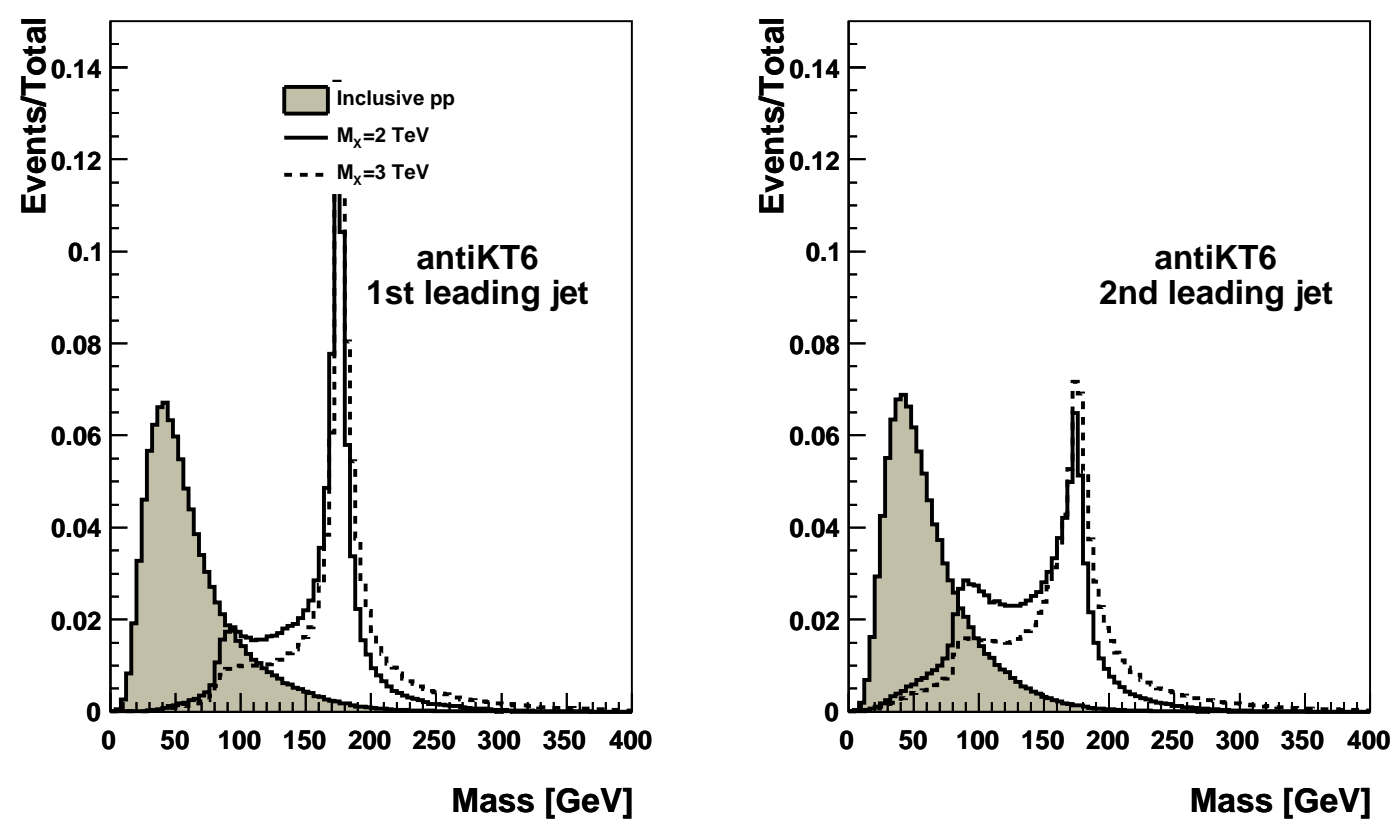

Figure 4: The jet masses for the first (left) and second (right) leading jets in $p_{T}$ reconstructed using the anti- $k_{T}$ jet algorithm with $R=0.6$. 
set to decay hadronically. The PYTHIA parameters were set to the default ATLAS parameters tuned to describe multiple interactions [24]. The jets were reconstructed using the anti- $k_{T}$ algorithm using the final-state hadrons. The events were first generated and stored for easy processing.

Figure 2 shows the jet-shape variables for: (1) inclusive $p p$ production and (2) for $Z^{\prime}$, with a mass of 2 and $3 \mathrm{TeV}$ (solid and dashed lines, respectively). Events were selected with at least one jet with transverse momentum $p_{T}($ jet $)>500 \mathrm{GeV}$ and within the pseudorapidity range $|\eta(j e t)|<2.5$. The histograms show the jet shape variables using fully inclusive QCD events with an identical event selection.

It is evident that all jet shapes are rather different for inclusive $p p$ collisions and collisions involving the production of TeV-scale particles decaying into $t \bar{t}$. The observed differences between the two processes can easily be understood. Jets originating from top decays have masses close to the top-quark mass (for a complete overlap of decay products) or $W$ mass. Such jets are wider and are more elongated than standard QCD jets due to the decay plane formed by the channel $t \rightarrow W b$ (eccentricity values are shifted to unity).

The jet mass for the second leading jet in $p_{T}$ is shown in Fig. 3 . The peak of this distribution is shifted towards the $W$ mass. Other jet-shape variables are rather similar to those shown in Fig. 2,

The use of the anti- $k_{T}$ algorithm with a large separation parameter $R$ is expected to be more efficient since hadrons from top decays will have a higher chance to be contained inside single monojets. We have calculated the jet shapes for the anti- $k_{T}$ algorithm with $R=0.6$. The largest difference with respect to the case $R=0.4$ is for the jet masses as shown in Fig. 4, The $W$ mass peak shown in Fig. 2(a) is significantly reduced for jets with $R=0.6$. At the same time, the peak attributed to the top quarks is more pronounced. This is not surprising since a jet cone of 0.6 is more likely to fully contain the decay products. The conclusion is that the anti- $k_{T}$ jets with $R=0.6$ are better suited for the searches using dijet invariant masses and the jet-shape variables, since they allow a better separation of the signal events from the QCD-induced background.

With the power of jet-shape variables in our hands, one can proceed with the rejection of QCD-induced jets in searches for TeV-mass particles. For simplicity, we apply a simple cut method, i.e. we will place cuts on the jet-shape variables to reject QCD background events. More elaborate studies will likely require the maximumlikelihood approach or a construction of a neural network for event rejection. We also concentrate on the anti- $k_{T}$ algorithm with the size 0.4 as shown in Figs. 2-3. The following selection cuts were used:

- $M($ jet $)>70 \mathrm{GeV}$, with $M($ jet $)$ being the jet mass;

- $W($ jet $)>0.07$, with $W($ jet $)$ being the jet width;

- $\mathrm{EEC}>0.75$. 
The above cuts are indicated on Fig. 2. We applied identical cuts on the first and the second leading in $p_{T}$ jet, although the cuts should be optimized depending on the average transverse momentum. We also did not tune our cuts for the $3 \mathrm{TeV}$ mass range; these should be somewhat looser in order to achieve the best possible efficiency for the reconstruction of $X$ particles.

It has already been discussed that $b$-tagging information can help in background reduction [2]. We did not use $b$-tagging information for our calculation, since its inclusion requires a detailed knowledge of experimental efficiencies and purities. These will clearly be affected by the potentially large boost of the $b$-quarks and thus a very detailed experimental study of this problem is necessary.

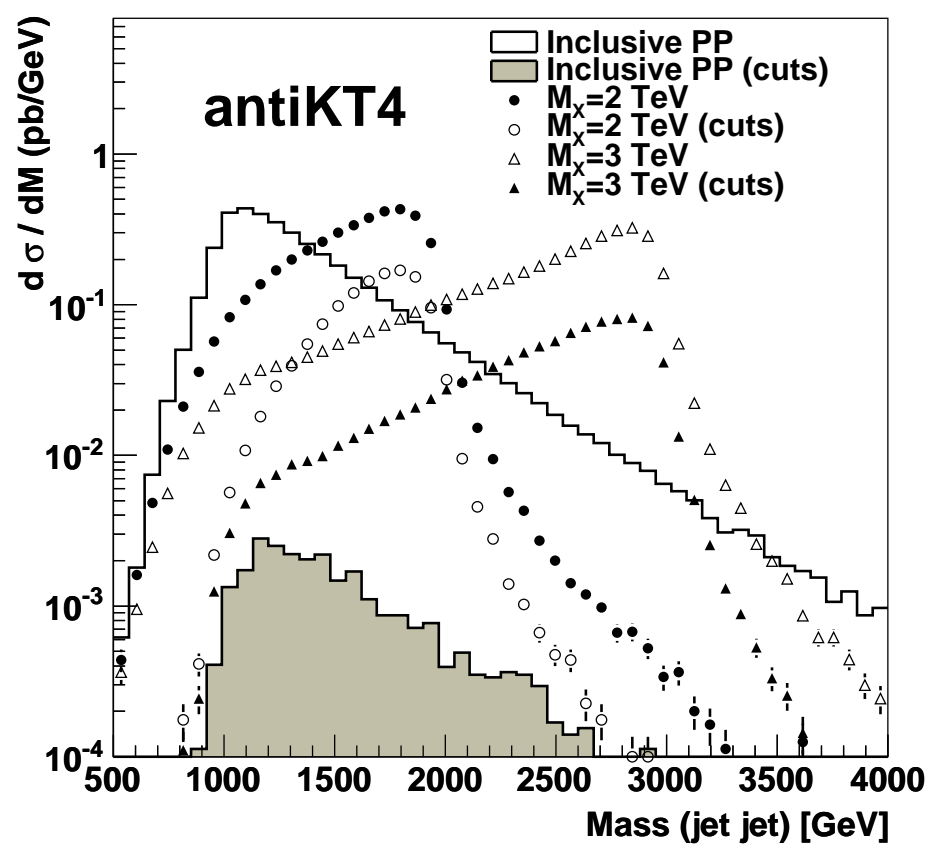

Figure 5: The jet-jet invariant mass before and after the applied event-selection cuts described in the text. The signal events (before the selection cuts) for particles with the masses 2 and $3 \mathrm{TeV}$ are normalized to the background events.

Figure 5 shows the jet-jet invariant mass before and after the applied cuts. For illustration, the distribution with the signal events before the selection cuts was normalized to the total number of background events. The rejection factor for QCD events in the mass range $1.5-2 \mathrm{TeV}$ is roughly 105 , while it is only a factor of 2.7 for the signal events. Therefore, the ratio of the rejection factors for inclusive QCD and events with heavy states is about 38. A similar conclusion was obtained for 3 $\mathrm{TeV}$ particles, although the rejection of the signal events was somewhat larger (3.7) since no attempt was made to fine tune our cuts for this mass range. 
Figure [6] shows the jet-jet invariant mass for an integrated luminosity of $200 \mathrm{pb}^{-1}$ at $10 \mathrm{TeV}$ center-of-mass energy. The distribution is shown after the event-selection cuts described above. The shaded histogram shows the expected QCD background alone, while the solid symbols show QCD inclusive events plus contributions of 2 $\mathrm{TeV}$ (open symbols) and $3 \mathrm{TeV}$ (open triangles) particles. The number of the signal events was determined in each case such that the overall signal-plus-background distribution features two bumps with $6-7 \sigma$ significance level. The statistical significance of such enhancements was estimated using a $\chi^{2}$ fit with a Gaussian plus second-order polynomial function.

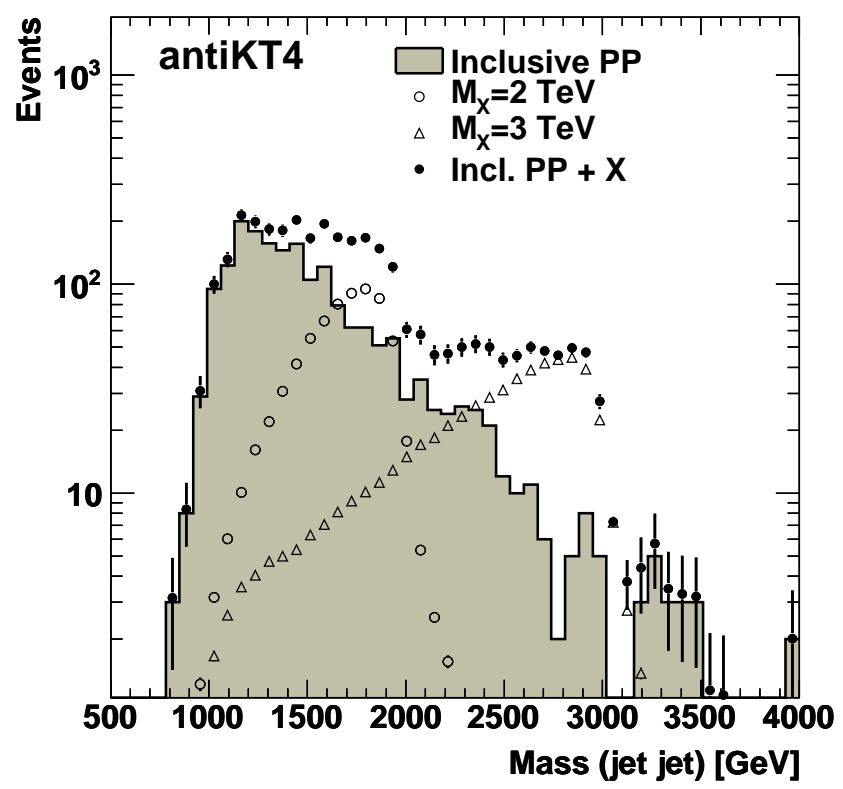

Figure 6: Jet-jet mass as in Fig. [5, but the distribution is presented in terms of the number of events for $200 \mathrm{pb}^{-1}$ of integrated luminosity. Also shown is the fraction of signal events necessary for a $6 \sigma$ observation of a broad peak in the jet-jet invariant mass.

From the number of the signal events shown in Figure 6, one can calculate the cross section $\sigma(X) \operatorname{Br}(X \rightarrow t \bar{t})$ for a discovery of a new TeV-mass particle at the $6 \sigma$ confidence level. For a $2 \mathrm{TeV}$ mass, this cross section is about $2-3 \mathrm{pb}$. This cross section can be as low as $1 \mathrm{pb}$ for $3 \mathrm{TeV}$ particles.

In comparison with other discovery channels, the obtained cross section for a $6 \sigma$ observation is an order of magnitude smaller than that expected for the SM Higgs boson with the mass $150 \mathrm{GeV}$, and roughly equals to the production cross section for Higgs bosons with a mass $700 \mathrm{GeV}$ [25]. 


\section{Discussion}

Although the goal of this paper is to extract the discovery-level cross section for $t \bar{t}$ resonances using jet-shape techniques, it is natural to ask how the approach discussed in this paper is different from other publications based on jet substructure.

A rejection factor 100 for QCD inclusive events was achieved in Ref. [6] using subjets in fully-hadronic $t \bar{t}$ decays and then imposing kinematic constraints. The Cambridge-Aachen jet clustering algorithm [26] was used in this approach which uses features of this algorithm to identify jets with substructure by decomposing jets into subjets. A similar rejection factor was achieved by the CMS Collaboration [11] also using the Cambridge-Aachen jet clustering algorithm after decomposing jets into subjets and examining kinematics of these subjets. Another closely related study has recently been performed by the ATLAS collaboration [10] with the goal to improve the rejection efficiency discussed in their previous publication [7, 14]. While the jet substructure was used for the monojet, the main improvement in rejection of QCD background rejection comes from requiring semi-leptonic decays.

The approach used in this paper does not include kinematic constraints on subjet kinematics, since we do not attempt to resolve kinematic characteristics of separate subjets inside jets. Instead, this paper relies primarily on the jet shapes (jet width and eccentricity) and jet masses. Thus the details of jet fine-structure are less important and, as a result, this method is expected to have smaller sensitivity to the fragmentation mechanism and to detector effects (such as calorimeter segmentation). Moreover, this approach is based on the anti- $k_{T}$ algorithm which is expected to have an advantage in events with pile-up.

\section{Summary}

We have shown that the jet-shape approach is extremely powerful for reduction of the contribution from QCD jets to dijet invariant masses which can be used for searches of $\mathrm{TeV}$-scale particles. A rejection factor above 100 can easily be achieved for inclusive $p p$ collisions, with only a factor three reduction for the signal events for a $2 \mathrm{TeV}$ particle decaying to $t \bar{t}$ using jets reconstructed with the anti- $k_{T}$ algorithm with the size $R=0.4$. This was obtained without sophisticated reconstruction methods, without careful tuning of cuts for both leading in $p_{T}$ jets and without using $b$-tagging information. This rejection factor is a factor of four larger compared to the case without using jet-shape information [2] and similar to that obtained in [11].

It should be noted that a full detector simulation may change the values of the rejection factors. However, no large change in our conclusions is expected since jets at the transverse momenta considered in this paper are typically well reconstructed.

The approach discussed in this paper is illustrated using the $X \rightarrow t \bar{t} \rightarrow W^{+} b_{1} W^{-} b_{2}$ decay channel, with subsequent hadronic decays of the $W$ mesons. Decay channels involving $t \bar{t}$ are the most promising since they involve top quarks that are known 
couple most strongly to the electroweak symmetry breaking sector. However, we expect a similar conclusion for other cascade-type decays.

The jet-shape approach allows to obtain a very competitive $\sigma(X) \operatorname{Br}(X \rightarrow t \bar{t}) \simeq$ $2-3 \mathrm{pb}$ cross section for a $6 \sigma$ observation of an enhancement in jet-jet invariantmass spectra in the mass range 2-3 TeV. As example, this cross section is one order of magnitude smaller than that expected for the SM Higgs production with the mass $150 \mathrm{GeV}$ at a similar center-of-mass energies.

\section{Acknowledgements}

We would like to thank Brian Martin (MSU) and Gavin Salam (LPTHE) for useful discussions of the anti- $k_{T}$ algorithm and the FastJet package.

\section{References}

[1] K. Agashe, A. Belyaev, T. Krupovnickas, G. Perez, J. Virzi, Phys. Rev. D77 (2008) 015003.

[2] B. Lillie, L. Randall, L.-T. Wang, JHEP 09 (2007) 074.

[3] J. M. Butterworth, J. R. Ellis, A. R. Raklev, JHEP 05 (2007) 033.

[4] L. G. Almeida, S. J. Lee, G. Perez, I. Sung, J. Virzi, Phys. Rev. D79 (2009) 074012.

[5] L. G. Almeida, et al., Phys. Rev. D79 (2009) 074017.

[6] D. E. Kaplan, K. Rehermann, M. D. Schwartz, B. Tweedie, Phys. Rev. Lett. 101 (2008) 142001.

[7] G. H. Brooijmans, High pT Hadronic Top Quark Identification. Published in "A Les Houches Report. Physics at Tev Colliders 2007 - New Physics Working Group", Preprint hep-ph/0802.3715, 2008.

[8] J. M. Butterworth, et al., Discovering baryon-number violating neutralino decays at the LHC, Preprint CERN-PH-TH/2009-073, hep-ph/0906.0728, 2009.

[9] S. D. Ellis, C. K. Vermilion, J. R. Walsh, Phys. Rev. D80 (2009) 051501.

[10] ATLAS Collaboration, Reconstruction of High Mass t $\bar{t}$ Resonances in the Lepton+Jets Channel, Technical Report ATL-PHYS-PUB-2009-081. ATL-COMPHYS-2009-255, CERN, Geneva, May 2009.

[11] CMS Collaboration, A Cambridge-Aachen ( $C$-A) based Jet Algorithm for boosted top-jet tagging, Technical Report CMS-PAS-JME-09-001, Jul 2009. 
[12] S. Matsumoto, M. M. Nojiri, D. Nomura, Phys. Rev. D75 (2007) 055006.

[13] A. L. Fitzpatrick, J. Kaplan, L. Randall, L.-T. Wang, JHEP 09 (2007) 013.

[14] G. H. Brooijmans, et al., New Physics at the LHC: A Les Houches Report. Physics at Tev Colliders 2007 - New Physics Working Group, Preprint hep-ph/0802.3715, 2008.

[15] J. Thaler, L.-T. Wang, JHEP 07 (2008) 092.

[16] M. Cacciari, G. P. Salam, G. Soyez, JHEP 04 (2008) 063.

[17] M. Beneke, et al., Top quark physics, Preprint hep-ph/0003033, 2000.

[18] S. Catani, Y. L. Dokshitzer, M. H. Seymour, B. R. Webber, Nucl. Phys. B406 (1993) 187;

S. D. Ellis, D. E. Soper, Phys. Rev. D48 (1993) 3160.

[19] I. Jolliffe, Principal Component Analysis. Springer, 2002.

[20] T. Sjostrand, S. Mrenna, P. Z. Skands, JHEP 05 (2006) 026.

[21] S. Chekanov, Comput. Phys. Commun. 173 (2005) 175. Available on http://projects.hepforge.org/runmc/.

[22] I. Antcheva, et al., Comput. Phys. Commun. 180 (2009) 2499.

[23] M. Cacciari, G. Salam, G. Soyez, FastJet. A $C++$ library for the $k_{T}$ algorithm,

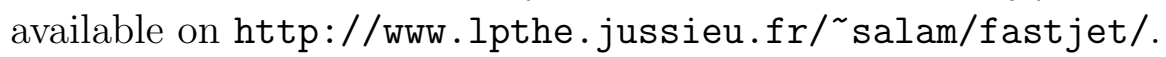

[24] ATLAS Collaboration, A. Moraes, Modeling the underlying event: Generating predictions for the LHC. ATL-PHYS-PROC-2009-045.

[25] C. Buttar, et al., Les houches physics at TeV colliders 2005, standard model and Higgs working group: Summary report, Preprint hep-ph/0604120, 2006.

[26] Y. L. Dokshitzer, G. D. Leder, S. Moretti, B. R. Webber, JHEP 08 (1997) 001. 